\title{
Terminating Non-Disjoint Combined Unification
}

\author{
Serdar Erbatur $^{1}$, Andrew M. Marshall ${ }^{2}$, and Christophe Ringeissen ${ }^{3}$ \\ 1 University of Texas at Dallas, USA \\ 2 University of Mary Washington, USA \\ 3 Université de Lorraine, CNRS, Inria, LORIA, F-54000 Nancy, France
}

\begin{abstract}
The equational unification problem, where the underlying equational theory may be given as the union of component equational theories, appears often in practice in many fields such as automated reasoning, logic programming, declarative programming, and the formal analysis of security protocols. In this paper, we investigate the unification problem in the non-disjoint union of equational theories via the combination of hierarchical unification procedures. In this context, a unification algorithm known for a base theory is extended with some additional inference rules to take into account the rest of the theory. We present a simple form of hierarchical unification procedure. The approach is particularly well-suited for any theory where a unification procedure can be obtained in a syntactic way using transformation rules to process the axioms of the theory. Hierarchical unification procedures are exemplified with various theories used in protocol analysis. Next, we look at modularity methods for combining theories already using a hierarchical approach. In addition, we consider a new complexity measure that allows us to obtain terminating (combined) hierarchical unification procedures.
\end{abstract}

\section{Introduction}

Unification is a critical tool in many fields such as automated reasoning, logic programming, declarative programming, and the formal analysis of security protocols. For many of these applications we want to consider equational unification, where the problem is defined modulo an equational theory $E$, such as Associativity-Commutativity. For example, one approach to the analysis of security protocols is based on deductive reasoning, as is done in the following tools 6 6 5 18 25. In this approach protocols are usually represented by clauses in first-order logic with equality and equational theories are used to specify the capabilities of an intruder [1. To support this reasoning approach we need to use $E$-unification procedures. Since equational unification is undecidable in general, specialized techniques have been developed to solve the problem for particular classes of equational theories, many of high practical interest. For instance, when the equational theory $E$ has the Finite Variant Property (FVP) [11]19], there exists a reduction from $E$-unification to syntactic unification via the computation of finitely many variants of the unification problem. The class of equational theories with the FVP has attracted a considerable interest since it contains theories that are crucial in protocol analysis [19|8|7|12|26]. 
Another ubiquitous scenario is given by an equational theory $E$ involved in a union of theories $F \cup E$. To solve this case, it is quite natural to proceed in a modular way by reusing the unification algorithms available for $F$ and for $E$. There are terminating and complete combination procedures for signaturedisjoint unions of theories [29/3. However, the non-disjoint case remains a challenging problem. One approach to the non-disjoint combination problem that has been successful in some cases is the hierarchical approach [14. In this approach, $F \cup E$-unification can be considered as a conservative extension of $E$-unification. Then, a new inference system related to $F$, say $U_{F}$, can be combined with an $E$-unification algorithm to obtain an $F \cup E$ unification algorithm. While this hierarchical approach won't work for every $F \cup E$ it can be a very useful tool when applicable. However, up to now it could be complex to know if a combination $F \cup E$ could be solved via the hierarchical approach. For example, there is no general method for obtaining the inference system $U_{F}$, and the resulting hierarchical unification procedure may not terminate.

In this paper, we consider "syntactic" theories $F \cup E$ where $U_{F}$ can be defined as a system of mutation rules, and we present new terminating instances of the hierarchical unification procedure. When an equational theory fulfills the syntacticness property 22 28, there exists a rule-based unification procedure in the same vein as the one known for syntactic unification, which is called a mutation-based unification procedure. Unfortunately, being syntactic is not a sufficient condition to ensure the termination of this mutation-based unification procedure. However, terminating mutation-based unification procedures are known for some particular theories such as one one-side distributivity [30|24, distributive exponentiation theories [15], shallow theories [10] and theories closed by paramodulation [23]. All the theories investigated here using the hierarchical approach are both syntactic and finitary: each of them is actually a syntactic theory for which a (finitary) unification algorithm is shown. On the one hand, we study theories which are both collapse-free and finitary, that is, finitary theories defined by axioms between non-variable terms. These theories are known to be syntactic [22]. On the other hand, we also examine forward-closed theories that are known to be both syntactic and finitary, just like theories closed by paramodulation 23. The forward-closed theories we are interested in are actually examples of theories having the Finite Variant Property.

The contributions of the paper consist of several improvements to the hierarchical combination method [14 13] including: simplifying the method, clarifying the theories for which the approach is applicable, and reducing some of the restrictions. Furthermore, we develop several new results including general reduction procedures for certain types of theories, and modular termination results. More specifically:

- We better define theories for which a hierarchical approach is applicable, constructor-based theories, and simplify the hierarchical unification procedure denoted here by $H_{E}\left(U_{F}\right)$, where $U_{F}$ is an additional rule-based procedure to be combined with an $E$-unification algorithm (Section 3 ). 
- We define the requirements for the $U_{F}$ rule-based procedure, and develop new general rule-based procedures for subterm collapse-free and forwardclosed theories (Section 3).

- Using the hierarchical approach, we develop new modularity results for the unification problem in unions of constructor-sharing theories. We define a new complexity measure to show terminating combinations of hierarchical unification algorithms. This allows us to obtain new (combined) unification algorithms for a wider variety of theories (Section 4).

- We show how the combination of hierarchical unification algorithms can be applied to unions of constructor-sharing forward-closed theories (Section 4).

The rest of the paper is organized as follows. Section 2 provides the background material. Section 2.3 contains an introduction to forward-closed theories. Section 3 introduces the notion of hierarchical unification and presents examples of theories admitting a hierarchical unification algorithm. Section 4 focuses on the combination of hierarchical unification algorithms. Finally, Section 5 contains the conclusions and future work.

\section{Preliminaries}

We use the standard notation of equational unification 4 and term rewriting systems [2]. Given a first-order signature $\Sigma$ and a (countable) set of variables $V$, the set of $\Sigma$-terms over variables $V$ is denoted by $T(\Sigma, V)$. The set of variables in a term $t$ is denoted by $\operatorname{Var}(t)$. A term $t$ is ground if $\operatorname{Var}(t)=\emptyset$. A term is linear if all its variables occur only once. For any position $p$ in a term $t$ (including the root position $\epsilon), t(p)$ is the symbol at position $p,\left.t\right|_{p}$ is the subterm of $t$ at position $p$, and $t[u]_{p}$ is the term $t$ in which $\left.t\right|_{p}$ is replaced by $u$. A substitution is an endomorphism of $T(\Sigma, V)$ with only finitely many variables not mapped to themselves. A substitution is denoted by $\sigma=\left\{x_{1} \mapsto t_{1}, \ldots, x_{m} \mapsto t_{m}\right\}$, where the domain of $\sigma$ is $\operatorname{Dom}(\sigma)=\left\{x_{1}, \ldots, x_{m}\right\}$. Application of a substitution $\sigma$ to $t$ is written $t \sigma$.

\subsection{Equational Theories}

Given a set $E$ of $\Sigma$-axioms (i.e., pairs of $\Sigma$-terms, denoted by $l=r$ ), the equational theory $=_{E}$ is the congruence closure of $E$ under the law of substitutivity (by a slight abuse of terminology, $E$ is often called an equational theory). Equivalently, $=_{E}$ can be defined as the reflexive transitive closure $\leftrightarrow_{E}^{*}$ of an equational step $\leftrightarrow_{E}$ defined as follows: $s \leftrightarrow_{E} t$ if there exist a position $p$ of $s, l=r$ (or $r=l$ ) in $E$, and substitution $\sigma$ such that $\left.s\right|_{p}=l \sigma$ and $t=s[r \sigma]_{p}$. An axiom $l=r$ is regular if $\operatorname{Var}(l)=\operatorname{Var}(r)$. An axiom $l=r$ is linear (resp., collapse-free) if $l$ and $r$ are linear (resp. non-variable terms). An equational theory is regular (resp., linear/collapse-free) if all its axioms are regular (resp., linear/collapse-free). A theory $E$ is subterm collapse-free if and only if for all terms $t$ it is not the case that $t={ }_{E} u$ where $u$ is a strict subterm of $t$. A theory $E$ is syntactic if it has 
finite resolvent presentation $S$, defined as a finite set of axioms $S$ such that each equality $t={ }_{E} u$ has an equational proof $t \leftrightarrow{ }_{S}^{*} u$ with at most one equational step $\leftrightarrow_{S}$ applied at the root position. One can easily check that $C=\{x * y=y * x\}$ (Commutativity) and $A C=\{x *(y * z)=(x * y) * z, x * y=y * x\}$ (AssociativityCommutativity) are regular, collapse-free, and linear. Moreover, $C$ and $A C$ are syntactic 22. A $\Sigma$-equation is a pair of $\Sigma$-terms denoted by $s={ }^{?} t$ or simply $s=t$ when it is clear from the context that we do not refer to an axiom. A flat $\Sigma$-equation is either an equation between variables or a non-variable flat $\Sigma$ equation of the form $x_{0}=f\left(x_{1}, \ldots, x_{n}\right)$ where $x_{0}, x_{1}, \ldots, x_{n}$ are variables and $f$ is a function symbol in $\Sigma$. An $E$-unification problem is a set of $\Sigma$-equations, $G=\left\{s_{1}=? t_{1}, \ldots, s_{n}=? t_{n}\right\}$, or equivalently a conjunction of $\Sigma$-equations. The set of variables in $G$ is denoted by $\operatorname{Var}(G)$. A solution to $G$, called an E-unifier, is a substitution $\sigma$ such that $s_{i} \sigma={ }_{E} t_{i} \sigma$ for all $1 \leq i \leq n$, written $E \models G \sigma$. A substitution $\sigma$ is more general modulo $E$ than $\theta$ on a set of variables $V$, denoted as $\sigma \leq_{E}^{V} \theta$, if there is a substitution $\tau$ such that $x \sigma \tau={ }_{E} x \theta$ for all $x \in V$. A Complete Set of E-Unifiers of $G$, denoted by $C S U_{E}(G)$, is a set of substitutions such that each $\sigma \in C S U_{E}(G)$ is an $E$-unifier of $G$, and for each $E$-unifier $\theta$ of $G$, there exists $\sigma \in C S U_{E}(G)$ such that $\sigma \leq_{E}^{\operatorname{Var}(G)} \theta$. An E-unification algorithm is an algorithm that computes a finite $C S U_{E}(G)$ for all $E$-unification problems $G$. An inference rule $G \vdash G^{\prime}$ for $E$-unification is sound if each $E$-unifier of $G^{\prime}$ is an $E$-unifier of $G$; and complete if for each $E$-unifier $\sigma$ of $G$, there exists an $E$-unifier $\sigma^{\prime}$ of $G^{\prime}$ such that $\sigma^{\prime} \leq_{E}^{\operatorname{Var}(G)} \sigma$. An inference system for $E$-unification is sound if all its inference rules are sound; and complete if for each $E$-unification problem $G$ on which an inference applies and each $E$-unifier $\sigma$ of $G$, there exist an $E$-unification problem $G^{\prime}$ inferred from $G$ and an $E$-unifier $\sigma^{\prime}$ of $G^{\prime}$ such that $\sigma^{\prime} \leq_{E}^{\operatorname{Var}(G)} \sigma$. A set of equations $G=\left\{x_{1}=? t_{1}, \ldots, x_{n}={ }^{?} t_{n}\right\}$ is said to be in tree solved form if each $x_{i}$ is a variable occurring once in $G$. Given an idempotent substitution $\sigma=\left\{x_{1} \mapsto t_{1}, \ldots, x_{n} \mapsto t_{n}\right\}$ (such that $\sigma \sigma=\sigma$ ), $\hat{\sigma}$ denotes the corresponding tree solved form. A set of equations is said to be in dag solved form if they can be arranged as a list $x_{1}=?{ }^{?} t_{1}, \ldots, x_{n}={ }^{?} t_{n}$ where (a) each left-hand side $x_{i}$ is a distinct variable, and (b) $\forall 1 \leq i \leq j \leq n$ : $x_{i}$ does not occur in $t_{j}$. A set of equations $\left\{x_{1}={ }^{?} t_{1}, \ldots, x_{n}=?{ }^{?} t_{n}\right\}$ is a cycle if for any $i \in[1, n-1], x_{i+1} \in \operatorname{Var}\left(t_{i}\right), x_{1} \in \operatorname{Var}\left(t_{n}\right)$, and there exists $j \in[1, n]$ such that $t_{j}$ is not a variable. Given two disjoint signatures $\Sigma_{0}$ and $\Sigma_{1}$ and any $i=1,0, \Sigma_{i}$-terms (including the variables) and $\Sigma_{i}$-equations (including the equations between variables) are called $\Sigma_{i}$-pure. A term $t$ is called a $\Sigma_{i}$-rooted term if its root symbol is in $\Sigma_{i}$. An alien subterm of a $\Sigma_{i}$-rooted term $t$ is a $\Sigma_{j}$-rooted subterm $s(i \neq j)$ such that all superterms of $s$ are $\Sigma_{i}$-rooted. We define general E-unification as the unification problem in the equational theory obtained by extending $E$ with arbitrary free function symbols.

Given a $\Sigma_{0}$-theory $E$, a theory $F \cup E$ is a conservative extension of $E$ if $={ }_{F \cup E}$ and $=_{E}$ coincide on $\Sigma_{0}$-terms. When $F \cup E$ is a conservative extension of $E, E$-unification is said to be complete for solving the $\Sigma_{0}$-fragment of $F \cup E$ unification if for any $\Sigma_{0}$-pure $F \cup E$-unification problem $G$, any $C_{S} U_{E}(G)$ is a 
$C S U_{F \cup E}(G)$. If $F$ and $E$ have disjoint signatures, $E$-unification is known to be complete for solving the $\Sigma_{0}$-fragment of $F \cup E$-unification.

\subsection{Equational Term Rewrite Systems}

Given a signature $\Sigma$, an equational term rewrite system (TRS) $(R, E)$ over $\Sigma$ is defined by a $\Sigma$-theory $E$ and a finite set $R$ of oriented $\Sigma$-axioms called rewrite rules and of the form $l \rightarrow r$ such that $l, r$ are $\Sigma$-terms, $l$ is not a variable and $\operatorname{Var}(r) \subseteq \operatorname{Var}(l)$. A term $s$ rewrites to a term $t$ w.r.t $(R, E)$, denoted by $s \rightarrow_{R, E} t$, if there exist a position $p$ of $s, l \rightarrow r \in R$, and substitution $\sigma$ such that $s_{\mid p}={ }_{E} l \sigma$ and $t=s[r \sigma]_{p}$. The term $s_{\mid p}$ is called a redex. Given a TRS $(R, E)$, $\longleftrightarrow{ }_{R \cup E}$ denotes the symmetric relation $\leftarrow_{R, E} \cup \rightarrow_{R, E} \cup=_{E}$. A TRS $(R, E)$ is Church-Rosser modulo $E$ if $\longleftrightarrow_{R \cup E}^{*}$ is included in $\rightarrow_{R, E}^{*} \circ={ }_{E} \circ \leftarrow_{R, E}^{*}$. When ${ }_{E} \circ \rightarrow_{R, E} \circ=_{E}$ is terminating, the following properties are equivalent [20]:

1. $(R, E)$ is Church-Rosser modulo $E$,

2. for any terms $t, t^{\prime}, t \longleftrightarrow{ }_{R \cup E}^{*} t^{\prime}$ if and only if $t \downarrow={ }_{E} t^{\prime} \downarrow$, where $t \downarrow$ (resp., $t^{\prime} \downarrow$ ) denotes any normal form of $t$ (resp., $\left.t^{\prime}\right)$ w.r.t $(R, E)$.

A TRS $(R, E)$ is E-convergent if $=_{E} \circ \rightarrow_{R, E} \circ=_{E}$ is terminating and $(R, E)$ is Church-Rosser modulo $E$. Let $\Sigma_{0}$ be the subsignature of $\Sigma$ that consists of function symbols occurring in the axioms of $E$. An $E$-convergent TRS $(R, E)$ is said to be E-constructed if $\Sigma_{0} \cap\{l(\epsilon) \mid l \rightarrow r \in R\}=\emptyset$.

An $E$-convergent TRS $(R, E)$ is said to be subterm $E$-convergent if for any $l \rightarrow r \in R, r$ is either a strict subterm of $l$ or a constant. When $(R, E)$ is clear from the context, a normal form w.r.t $(R, E)$ is said to be normalized. A substitution $\sigma$ is normalized if, for every variable $x$ in the domain of $\sigma, x \sigma$ is normalized. An instance $l \sigma \rightarrow r \sigma$ of a rule $l \rightarrow r \in R$ is a right-reduced instance if $\sigma_{\mid \operatorname{Var}(r)}$ is normalized. A term $t$ is an innermost redex if no subterm of $t$ is a redex. An $E$-convergent TRS $(R, E)$ is $I R R$ if every innermost redex is $R, E$-reducible by a right-reduced instance of a rule in $R$. An $E$-convergent TRS $(R, E)$ is $I R 1$ if every innermost redex is $R, E$-reducible to a normal form in one step.

To simplify the notation, we often use tuples of terms, say $\bar{u}=\left(u_{1}, \ldots, u_{n}\right)$, $\bar{v}=\left(v_{1}, \ldots, v_{n}\right)$. Applying a substitution $\sigma$ to $\bar{u}$ is the tuple $\bar{u} \sigma=\left(u_{1} \sigma, \ldots, u_{n} \sigma\right)$. The tuples $\bar{u}$ and $\bar{v}$ are said to be $E$-equal, denoted by $\bar{u}=E \bar{v}$, if $u_{1}=_{E}$ $v_{1}, \ldots, u_{n}={ }_{E} v_{n}$. Similarly, $\bar{u} \rightarrow_{R}^{*} \bar{v}$ if $u_{1} \rightarrow_{R}^{*} v_{1}, \ldots, u_{n} \rightarrow_{R}^{*} v_{n}, \bar{u}$ is normalized if $u_{1}, \ldots, u_{n}$ are normalized, and $\bar{u}=?$ ? $\bar{v}$ is $u_{1}=?{ }^{?} v_{1} \wedge \cdots \wedge u_{n}=? v_{n}$.

\subsection{Forward Closure}

In this section, we introduce the notion of finite forward closure, following the definition given in [21]. Consider the rule:

ForwardOverlap $g \rightarrow d\left[l^{\prime}\right], l \rightarrow r \vdash(g \rightarrow d[r]) \sigma$ where $g \rightarrow d\left[l^{\prime}\right], l \rightarrow r \in R, l^{\prime}$ is not a variable and $\sigma \in C S U_{E}\left(l^{\prime}=? l\right)$ 
For this inference rule, the notion of redundancy is defined with respect to an ordering on terms. We assume the existence of a simplification ordering $>$ such that $>$ is $E$-compatible, meaning that $s^{\prime}={ }_{E} s>t={ }_{E} t^{\prime}$ implies $s^{\prime}>t^{\prime}$, and $l>r$ for any $l \rightarrow r \in R$. ForwardOverlap is said to be redundant in $(R, E)$ if for each $g^{\prime}$ such that $g^{\prime}={ }_{E} g \sigma, g^{\prime}$ is $R, E$-reducible by a right-reduced instance $s \mu \rightarrow t \mu$ of $R$ and either $s \mu<g \sigma$ or $\left(s \mu={ }_{E} g \sigma\right.$ and $\left.t \mu<d\left[l^{\prime}\right] \sigma\right)$.

Let $\mathcal{I}$ be an inference system generating rewrite rules and whose inferences are possibly redundant, like for instance $\mathcal{I}=\{$ ForwardOverlap $\}$. Given an equational TRS $(R, E)$, the saturation of $(R, E)$ with respect to $\mathcal{I}$ is inductively defined as follows:

$-S_{\mathcal{T}}^{0}(R)=R$

- $S_{\mathcal{I}}^{k+1}(R)=S_{\mathcal{I}}^{k}(R) \cup\{\rho\}$ where the rule $\rho$ is obtained by applying an inference $i$ in $\mathcal{I}$ using $\left(S_{\mathcal{I}}^{k}(R), E\right)$ as equational TRS and such that $i$ is not redundant in $\left(S_{\mathcal{I}}^{k}(R), E\right)$.

Let $S_{\mathcal{I}}(R)=\bigcup_{k>0} S_{\mathcal{I}}^{k}(R)$. When $S_{\mathcal{I}}(R)$ is finite, $S_{\mathcal{I}}(R)$ is called a finite $\mathcal{I}$ saturation of $(R, \bar{E})$. An equational TRS $(R, E)$ is $\mathcal{I}$-saturated if $S_{\mathcal{I}}(R)=R$. An equational TRS has a finite forward closure if it has a finite $\mathcal{I}$-saturation for $\mathcal{I}=\{$ ForwardOverlap $\}$. An equational TRS is forward-closed if it is $\mathcal{I}$ saturated for $\mathcal{I}=\{$ ForwardOverlap $\}$.

Example 1. Any subterm E-convergent TRS has a finite forward closure. Subterm convergent TRSs are often used in the verification of security protocols [1, e.g., $\{\operatorname{dec}(\operatorname{enc}(x, y), y) \rightarrow x\}$ and $\{f \operatorname{st}(\operatorname{pair}(x, y)) \rightarrow x, \operatorname{snd}(\operatorname{pair}(x, y)) \rightarrow y\}$. The equational TRSs $\{\operatorname{dec}(\operatorname{enc}(x, k), k * y) \rightarrow x\}$ and $\{r m(x * k, k) \rightarrow x\}$ are subterm $E$-convergent for $E=A C(*)=\{x *(y * z)=(x * y) * z, x * y=y * x\}$.

Forward closure can be connected to the notion of Finite Variant Property (FVP, for short) introduced in 11. Given an $E$-convergent $\operatorname{TRS}(R, E)$, an $(R, E)$-variant of a term $t$ is a pair $((t \theta) \downarrow, \theta)$ where $\theta$ is a normalized substitution whose domain is included in $\operatorname{Var}(t) .(R, E)$ has the FVP if for any term $t$ there exists a finite set $V$ of $(R, E)$-variants of $t$ such that any $(R, E)$-variant of $t$ is componentwise $E$-equal to an instance of some element in $V$. If $(R, E)$ has the FVP, then any $R \cup E$-unification problem $G$ reduces to $E$-unification problems via the computation of finitely many variants of $G$ (viewed as a term with additional symbols). This computation can be performed using folding variant narrowing [19 12. In [7, it was shown that for any TRS $R, R$ has the FVP iff it has a finite forward closure. A similar equivalence holds for $E$-constructed TRSs:

Lemma 1. Assume $(R, E)$ is any $E$-constructed $T R S$ and $E$ is any regular and collapse-free equational theory such that E-unification is finitary. Then, $(R, E)$ has a finite forward closure iff $(R, E)$ has the FVP.

Proof. We rely on some results that have been shown in 21 for an inference system $\mathcal{I}$ including ForwardOverlap plus an additional Parallel rule whose premises are $s \rightarrow t, l \rightarrow r \in R, v=u\left[l^{\prime}\right] \in E$ such that $l^{\prime}$ is a non-variable strict subterm of $u$ which is $E$-unifiable with $l$. The following statements are proved in [21]: 
- $(R, E)$ is IR1 iff $(R, E)$ is $\mathcal{I}$-saturated.

- If $(R, E)$ is $I R 1$ and $E$-unification is finitary, then $(R, E)$ has the FVP.

- If $(R, E)$ has the FVP, then $(R, E)$ has a finite $\mathcal{I}$-saturation.

When $(R, E)$ is $E$-constructed and $E$ is a regular and collapse-free equational theory, Parallel does not apply since a $\Sigma_{0}$-rooted term $l^{\prime}$ is not $E$-unifiable with a $\Sigma \backslash \Sigma_{0}$-rooted term $l$. Thus, $\mathcal{I}$-saturation reduces to forward closure, $\mathcal{I}$-satured means forward-closed, and the above statements can be reworded accordingly. To conclude the proof, notice that if $R^{\prime}$ is a finite forward closure of $(R, E)$, then $\left(R^{\prime}, E\right)$ is forward-closed and both $\left(R^{\prime}, E\right)$ and $(R, E)$ have the FVP.

In this paper, $(R, E)$ is assumed to be $E$-constructed and so the signature of $(R, E)$ necessarily includes a non-empty set of function symbols that do not occur in the axioms of $E$. Thus, this means that we actually need general $E$-unification, i.e., $E$-unification with free function symbols, instead of $E$-unification. Fortunately, when $E$ is regular and collapse-free, $E$-unification is finitary if and only if general $E$-unification is finitary. This equivalence is a consequence of a classical disjoint combination method for regular and collapse-free theories 31 that allows us to build a general E-unification algorithm as a combination of the syntactic unification algorithm and an $E$-unification algorithm.

From now on, the equational theory $E$ is always assumed to be regular and collapse-free when $(R, E)$ is $E$-constructed.

\section{Hierarchical Unification}

Consider now a union of theories $R \cup E$ where $E$ is regular and collapse-free and $(R, E)$ is assumed to be $E$-constructed. Thanks to this assumption, $R$ and $E$ are "sufficiently separated" and thus we can envision the problem of building an $R \cup E$-unification algorithm as a combination of two unification procedures: a mutation-based unification procedure processing some $R \cup E$-equalities, and an $E$-unification algorithm. The approach we will use for this problem is the hierarchical approach. Informally, the approach works as follows:

- The set of equations is processed to separate the terms over the shared signature, $\Sigma_{0}$, from terms over the non-shared one, $\Sigma \backslash \Sigma_{0}$.

- The mutation-based procedure is then used to simplify the $\Sigma \backslash \Sigma_{0}$-equations.

- The remaining equations over the shared signature $\Sigma_{0}$ are solved using the E-unification algorithm.

- The process can repeat. If the process terminates in a solved form then the problem is solvable and a unifier is produced.

A hierarchical unification procedure is parameterized by an $E$-unification algorithm and a mutation-based reduction procedure $U$. It applies some additional rules given in Figure 1: Coalesce, Split, Flatten, and VA are used to separate the terms, $U$ is used to simplify the $\Sigma \backslash \Sigma_{0}$-equations, and finally, Solve calls the $E$-unification algorithm. 


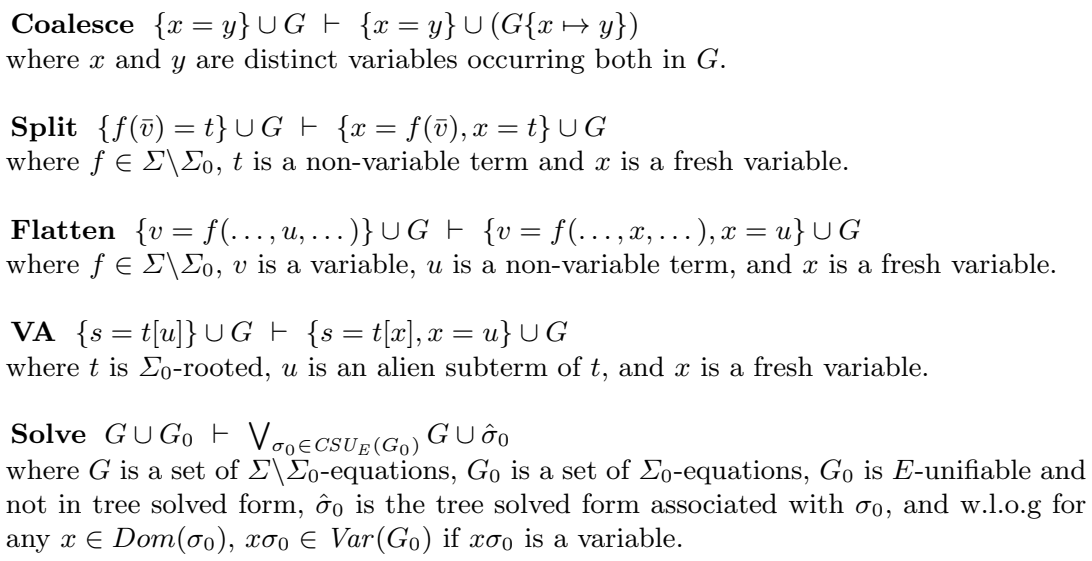

Fig. 1. $H_{E}$ rules

Definition 1 (Hierarchical unification procedure). Assume a $\Sigma_{0}$-theory $E$ for which an E-unification algorithm is known, a $\Sigma$-theory $F \cup E$ for which E-unification is complete for solving the $\Sigma_{0}$-fragment of $F \cup E$-unification, and an inference system $U$ such that: $U$ transforms only non-variable flat $\Sigma \backslash \Sigma_{0}$ equations; $U$ is sound and complete for $F \cup E$-unification; and $U$ is parameterized by some finite set $S$ of $F \cup E$-equalities for which the soundness of each inference $\vdash_{U}$ follows from at most one equality in $S$. Under these assumptions, $H_{E}(U)$ is the inference system defined as the repeated application of some inference from $H_{E}$ (cf. Figure 1) or U, using the following order of priority: Coalesce, Split, Flatten, VA, U, Solve. An F $\cup$ E-unification problem is separate, also called in separate form, if it is a normal form w.r.t $H_{E} \backslash\{$ Solve $\} . H_{E}(U)$ is said to be $a$ hierarchical unification procedure if the normal forms w.r.t $H_{E}(U)$ are either the separate dag solved forms or problems that are not $F \cup E$-unifiable.

Note that $U$ is not just a set of inference rules but also a strategy for applying those rules, for instance to avoid non-termination [15. From now on, an inference system $H_{E}(U)$ always denotes a hierarchical unification procedure.

Proposition 1. Let $(R, E)$ be any E-constructed TRS such that an inference system $U$ following Definition 1 is known for the equational theory $R \cup E$, in addition to an existing E-unification algorithm. Then $E, R \cup E$ and $U$ satisfy the assumptions of Definition 1, and a hierarchical unification procedure $H_{E}(U)$ provides a sound and complete $R \cup E$-unification procedure, and in particular an $R \cup$ E-unification algorithm when $H_{E}(U)$ is also terminating.

Proof. If $(R, E)$ is $E$-constructed, then $E$-unification is complete for solving the $\Sigma_{0}$-fragment of $R \cup E$-unification, and so all the assumptions are satisfied to define $H_{E}(U)$. By construction, $H_{E}(U)$ is sound and complete. Since the $R \cup E$ unifiable normal forms w.r.t $H_{E}(U)$ are assumed to be the separate dag solved forms, collecting all the separate dag solved forms reached by $H_{E}(U)$ suffices to get a complete set of $R \cup E$-unifiers. 


\subsection{Subterm Collapse-Free Theories}

Hierarchical unification algorithms are known for particular subterm collapsefree theories of particular interest for protocol analysis.

Proposition 2. (3015]) Let $E$ be the empty $\Sigma_{0}$-theory where $\Sigma_{0}$ only consists of a binary function symbol $*, R_{\mathcal{D}}=\{h(x * y) \rightarrow h(x) * h(y)\}$ and $R_{\mathcal{D} 1}=$ $\{f(x * y, z) \rightarrow f(x, z) * f(y, z)\}$. The equational TRSs $\left(R_{\mathcal{D}}, E\right)$ and $\left(R_{\mathcal{D} 1}, E\right)$ are E-constructed. Moreover, $R_{\mathcal{D}} \cup E$ (resp., $R_{\mathcal{D} 1} \cup E$ ) is a subterm collapse-free theory admitting a unification algorithm of the form $H_{E}\left(U_{\mathcal{D}}\right)\left(\right.$ resp., $\left.H_{E}\left(U_{\mathcal{D} 1}\right)\right)$.

Proof. Subterm collapse-freeness follows from the fact that both theories are non-size-reducing. The inference system $U_{\mathcal{D} 1}$ can be derived following the approach developed in 15 and based on the one initiated in 30 for one-side distributivity. The same approach can be applied for $R_{\mathcal{D}}$ to get $U_{\mathcal{D}}$.

Proposition 3. (15]) Let $A C=A C(\circledast), R_{\mathcal{E}}=\{\exp (\exp (x, y), z) \rightarrow \exp (x, y \circledast$ $z), \exp (x * y, z) \rightarrow \exp (x, z) * \exp (y, z)\}$ and $R_{\mathcal{F}}=\{\operatorname{enc}(\operatorname{enc}(x, y), z) \rightarrow \operatorname{enc}(x, y \circledast$ $z)\}$. The equational TRSs $\left(R_{\mathcal{E}}, A C\right)$ and $\left(R_{\mathcal{F}}, A C\right)$ are $A C$-constructed. Moreover, $\mathcal{E}_{A C}=R_{\mathcal{E}} \cup A C$ (resp., $\mathcal{F}_{A C}=R_{\mathcal{F}} \cup A C$ ) is a subterm collapse-free theory admitting a unification algorithm of the form $H_{A C}\left(U_{\mathcal{E}}\right)$ (resp., $H_{A C}\left(U_{\mathcal{F}}\right)$ ).

Proof. In [15] it is shown that both $\mathcal{E}_{A C}$ and $\mathcal{F}_{A C}$ are subterm collapse-free theories. Also in [15] a mutation-based inference system, say $U_{\mathcal{E}}$ (resp., $U_{\mathcal{F}}$ ), is developed for $\mathcal{E}_{A C}$ (resp., $\mathcal{F}_{A C}$ ): it reduces the $\Sigma \backslash \Sigma_{0}$-equations into solved forms after which a solving step applies $A C$-unification on $\Sigma_{0}$-equations. It is shown in [15] that the solving step needs only be applied once. Hence, the $\mathcal{E}_{A C^{-}}$ unification algorithm (resp., $\mathcal{F}_{A C}$-unification algorithm) given in [15] provides a unification algorithm of the form $H_{A C}\left(U_{\mathcal{E}}\right)$ (resp., $H_{A C}\left(U_{\mathcal{F}}\right)$ ).

\subsection{Forward-Closed E-Constructed TRSs}

For any forward-closed $E$-constructed TRS $(R, E)$ such that $E$ is regular and collapse-free, an $R \cup E$-unification algorithm of the form $H_{E}(U)$ can be obtained by defining some inference system $U$, based on the Basic Syntactic Mutation approach initiated for the class of theories closed by paramodulation [23], and already applied in [13] to a particular class of forward-closed equational TRSs.

Let $B S M_{R}$ be the inference system given in Figure 2. One can notice that each inference rule in $B S M_{R}$ generates some boxed terms. This particular annotation of terms, detailed in 2313, allows us to control the rules application, disregarding needless inferences on boxed terms, in such a way that $B S M_{R}$ is terminating.

An $R \cup E$-unification algorithm combining $B S M_{R}$ and an $E$-unification algorithm has been developed in [13] for the case of any forward-closed convergent TRS $R$ such that the left-hand sides of $R$ are linear and contain no symbols of $E$. In this paper, we extend 13 to any forward-closed $E$-constructed TRS $(R, E)$, without any further restriction on $R$.

The soundness and completeness of $B S M_{R}$ is shown by the following lemma. 
Imit $\bigcup_{i}\left\{x=f\left(\bar{v}_{i}\right)\right\} \cup G \vdash\{x=f(\bar{y})\} \cup \bigcup_{i}\left\{\bar{y}=\bar{v}_{i}\right\} \cup G$

where $f \in \Sigma \backslash \Sigma_{0}, i>1, \bar{y}$ are fresh variables and there are no more equations $x=f(\ldots)$ in $G$.

MutConflict $_{R}\{x=f(\bar{v})\} \cup G \vdash\{x=t, \bar{s}=\bar{v}\} \cup G$

where $f \in \Sigma \backslash \Sigma_{0}, f(\bar{s}) \rightarrow t$ is a fresh instance of a rule in $R, f(\bar{v})$ is unboxed, and there is another equation $x=u$ in $G$ with a non-variable term $u$ or $x=f(\bar{v})$ occurs in a cycle.

ImitCycle $\{x=f(\bar{v})\} \cup G \vdash\{x=f(\bar{y}), \bar{y}=\bar{v}\} \cup G$

where $f \in \Sigma \backslash \Sigma_{0}, f(\bar{v})$ is unboxed, $\bar{y}$ are fresh variables and $x=f(\bar{v})$ occurs in a cycle.

Fig. 2. $B S M_{R}$ rules

Lemma 2. Let $(R, E)$ be any forward-closed E-constructed TRS over the signature $\Sigma$. For each equality $u={ }_{R \cup E} v$ such that $u$ is $\Sigma \backslash \Sigma_{0}$-rooted and $v$ is normalized, one of the following is true:

1. $u=f(\bar{u}), v=f(\bar{v})$ and $\bar{u}={ }_{R \cup E} \bar{v}$.

2. $u=f(\bar{u})$, there exist $f(\bar{s}) \rightarrow t \in R$ and a normalized substitution $\sigma$ such that $\bar{u}={ }_{R \cup E} \bar{s} \sigma, v={ }_{E}$ t $\sigma$ and $\bar{s} \sigma, t \sigma$ are normalized.

Proof. Let us analyze the possible rewrite proofs $\rightarrow_{R, E}^{*}$ of $u={ }_{R \cup E} v$.

First, if there is no step at the root position, then we get $u=f(\bar{u}) \rightarrow_{R, E}^{*}$ $f\left(\bar{u}^{\prime}\right)=_{E} v$ where $\bar{u} \rightarrow_{R, E}^{*} \bar{u}^{\prime}$ and $\bar{u}^{\prime}$ are normalized. Since $f$ is a free symbol for $E$, we have that $v=f(\bar{v})$ and $\bar{u}^{\prime}={ }_{E} \bar{v}$. Hence, $\bar{u}={ }_{R \cup E} \bar{v}$ since $\bar{u}={ }_{R \cup E} \overline{u^{\prime}}$.

Second, if there is one step at the root position, then we have

$$
u=f(\bar{u}) \rightarrow_{R, E}^{*} f\left(\bar{u}^{\prime}\right)=f(\bar{s}) \sigma \rightarrow_{R, E, \epsilon} t \sigma={ }_{E} v
$$

where $f(\bar{s}) \rightarrow t \in R, \bar{u} \rightarrow_{R, E}^{*} \bar{u}^{\prime}, \bar{u}^{\prime}$ are normalized, $\bar{u}^{\prime}=_{E} \bar{s} \sigma$, and so $\sigma, \bar{s} \sigma$ are normalized. Since $t \sigma=_{E} v$ and $v$ is normalized, $t \sigma$ is also normalized.

A unification procedure of the form $H_{E}\left(B S M_{R}\right)$ corresponds to the $B S C$ unification procedure given in [13] except that Solve is applied in $B S C$ before $B S M_{R}$ rules. However, the termination proof stated for $B S C$ in [13] also holds when Solve is applied after the $B S M_{R}$ rules.

Lemma 3. Assume $E$ is any regular and collapse-free theory such that an $E$ unification algorithm is known. Let $(R, E)$ be a forward-closed E-constructed TRS and $B S M_{R}$ the inference system given in Fig. 2. Then $H_{E}\left(B S M_{R}\right)$ is an $R \cup$ E-unification algorithm.

Example 2. Consider $R=\{h(x) \rightarrow a \times x\}, R^{\prime}=\left\{f(x, y) \rightarrow a^{\prime}(y) \times x\right\}$ and $E=\{x \times(y * z)=(x \times y) *(x \times z)\}$. The theory $E$ corresponds to left-distributivity and an $E$-unification algorithm is given in [30. Since $(R, E)$ and $\left(R^{\prime}, E\right)$ are forward-closed and $E$-constructed, $H_{E}\left(B S M_{R}\right)$ and $H_{E}\left(B S M_{R^{\prime}}\right)$ are unification algorithms for $R \cup E$ and $R^{\prime} \cup E$, respectively. Notice that $h(x * y)={ }_{R \cup E} h(x) * h(y)$ and $f(x * y, z)={ }_{R^{\prime} \cup E} f(x, z) * f(y, z)$. 
Example 3. Consider $R=\left\{\pi_{1}(x . y) \rightarrow x, \pi_{2}(x . y) \rightarrow y, \operatorname{dec}(\operatorname{enc}(x, y), y) \rightarrow x\right\}$ and $E=\{e n c(x . y, z)=\operatorname{enc}(x, z) \cdot e n c(y, z)\}$. An $E$-unification algorithm can be obtained following the approach developed in [30[15] and can be used in a hierarchical unification procedure of the form $H_{E}\left(B S M_{R}\right)$. Since $(R, E)$ is forward-closed and $E$-constructed, $H_{E}\left(B S M_{R}\right)$ is an $R \cup E$-unification algorithm.

\section{Combined Hierarchical Unification}

We are now interested in combining hierarchical unification algorithms known for $E$-constructed TRSs. Given two $E$-constructed TRSs, say $\left(R_{1}, E\right)$ and $\left(R_{2}, E\right)$, the problem is to study the possible construction of a (combined) hierarchical unification algorithm for $\left(R_{1} \cup R_{2}, E\right)$ using the two hierarchical unification algorithms known for $\left(R_{1}, E\right)$ and $\left(R_{2}, E\right)$. We investigate this combination problem for the two classes of $E$-constructed TRSs introduced in Section 3 First, we consider a class of $E$-constructed TRSs $(R, E)$ such that $R \cup E$ is subterm collapse-free. Second, we study the class of forward-closed $E$-constructed TRSs $(R, E)$ such that $E$ is regular and collapse-free.

\subsection{Combining Subterm Collapse-Free Theories}

Let us first consider a technical lemma which is useful to get a hierarchical unification procedure.

Lemma 4. Let $\left(R_{1}, E\right)$ and $\left(R_{2}, E\right)$ be two E-constructed TRSs over the signatures $\Sigma_{1}$ and $\Sigma_{2}$, respectively, such that $\Sigma_{1} \cap \Sigma_{2}=\Sigma_{0}$ for the signature $\Sigma_{0}$ of $E$, and for $i=1,2, R_{i} \cup E$ admits a sound and complete unification procedure of the form $H_{E}\left(U_{i}\right)$. Assume that $R_{1} \cup R_{2} \cup E$ is subterm collapse-free, and for any $\Sigma_{1} \backslash \Sigma_{0}$-rooted term $t_{1}$ and any $\Sigma_{2} \backslash \Sigma_{0}$-rooted term $t_{2}, t_{1}$ cannot be equal to $t_{2}$ modulo $R_{1} \cup R_{2} \cup E$. Then, $H_{E}\left(U_{1} \cup U_{2}\right)$ is a sound and complete $R_{1} \cup R_{2} \cup$ E-unification procedure.

Proof. According to the assumptions, $U_{1} \cup U_{2}$ is sound and complete for $R_{1} \cup$ $R_{2} \cup E$-unification and any normal form w.r.t $H_{E}\left(U_{1} \cup U_{2}\right)$ is $R_{1} \cup R_{2} \cup E$-unifiable iff it is in dag solved form. So, Proposition 1 applies.

We study below a possible way to satisfy the assumptions of Lemma 4

Definition 2 (Layer-preservingness). Let $(R, E)$ be an E-constructed TRS over the signature $\Sigma$, for which $\Sigma_{0}$ denotes the signature of $E$. $A \Sigma$-term $t$ is said to be E-capped if there exist a constant-free $\Sigma_{0}$-term $u$ and a substitution $\sigma$ such that $t=u \sigma, \operatorname{Dom}(\sigma)=\operatorname{Var}(u)$ and $\operatorname{Ran}(\sigma)$ is a set of $\Sigma \backslash \Sigma_{0}$-rooted terms. The TRS $(R, E)$ is said to be layer-preserving if $R \cup E$ is subterm collapse-free and any normal form of any $\Sigma \backslash \Sigma_{0}$-rooted term is E-capped.

Remark 1. An easy way to get layer-preservingness of $(R, E)$ is to assume that $R \cup E$ is subterm collapse-free and the right hand-sides of rules in $R$ are $\Sigma \backslash \Sigma_{0^{-}}$ rooted. In that case the term $u$ in Definition 2 is simply a variable. Layerpreservingness generalizes this assumption used in [14]. 
The property of being $E$-constructed and layer-preserving is modular.

Lemma 5. Assume $E$ is a subterm collapse-free $\Sigma_{0}$-theory, for $i=1,2,\left(R_{i}, E\right)$ is an E-constructed layer-preserving TRS over the signature $\Sigma_{i}$, and $\Sigma_{1} \cap \Sigma_{2}=$ $\Sigma_{0}$. If $=_{E} \circ \rightarrow_{R_{1} \cup R_{2}} \circ=_{E}$ is terminating, then $\left(R_{1} \cup R_{2}, E\right)$ is an E-constructed layer-preserving TRS, and for any $\Sigma_{1} \backslash \Sigma_{0}$-rooted term $t_{1}$ and any $\Sigma_{2} \backslash \Sigma_{0}$-rooted term $t_{2}, t_{1}$ cannot be equal to $t_{2}$ modulo $R_{1} \cup R_{2} \cup E$.

Proof. To show that $\left(R_{1} \cup R_{2}, E\right)$ is layer-preserving, we have to prove that $R_{1} \cup R_{2} \cup E$ remains subterm collapse-free. The modularity of subterm collapsefreeness has been shown in [14] when the right-hand sides of $R_{i}$ are $\Sigma_{i} \backslash \Sigma_{0}$-rooted, for $i=1,2$. Actually, a similar proof by contradiction can be performed in the case $\left(R_{i}, E\right)$ is layer-preserving, for $i=1,2$. Let us consider the height of layers of a term $t$, inductively defined as follows:

$-h t(t)=0$ if $t$ is a variable,

$-h t(t)=1$ if $t$ is a non-variable pure term,

- $h t(t)=1+\max \{h t(u) \mid u$ is an alien subterm of $t\}$ if $t$ is not pure.

Assume there exists a term $t$ and a non-empty position $p$ such that $t={ }_{R_{1}} \cup E_{2} \cup E$ $t_{\mid p}$. If the path from $\epsilon$ to $p$ contains only symbols from one theory, say $R_{i} \cup E$, this would lead to a contradiction with the subterm collapse-freeness of $R_{i} \cup E$. Consider now that the path from $\epsilon$ to $p$ contains both a $\Sigma_{1} \backslash \Sigma_{0}$-symbol and a $\Sigma_{2} \backslash \Sigma_{0}$-symbol. Let $u=\left.t\right|_{p}$ and let $t^{\prime}$ and $u^{\prime}$ be the respective normal forms of $t$ and $u$ w.r.t $\left(R_{1} \cup R_{2}, E\right)$. Since $t^{\prime}=_{E} u^{\prime}$ and $E$ is necessarily regular collapse-free, we have that $t^{\prime}$ and $u^{\prime}$ have the same height of layers. By the layer-preserving assumption, $t$ and $t^{\prime}$ have the same height of layers, as well as $u$ and $u^{\prime}$. Thus $t$ and $u$ have the the same height of layers, which leads to a contradiction due to the considered path from $\epsilon$ to $p$.

Assume there exist some $\Sigma_{1} \backslash \Sigma_{0}$-rooted term $t_{1}$ and some $\Sigma_{2} \backslash \Sigma_{0}$-rooted term $t_{2}$ such that $t_{1}=R_{1} \cup R_{2} \cup E t_{2}$. Then, $t_{1}^{\prime}={ }_{E} t_{2}^{\prime}$ where $t_{1}^{\prime}$ and $t_{2}^{\prime}$ are the respective normal forms of $t_{1}$ and $t_{2}$ w.r.t $\left(R_{1} \cup R_{2}, E\right)$. The layer-preserving assumption implies that $t_{i}^{\prime}$ must still contain a symbol in $\Sigma_{i} \backslash \Sigma_{0}$ for $i=1,2$. Since $E$ is necessarily regular and collapse-free, it is thus impossible to have $t_{1}^{\prime}={ }_{E} t_{2}^{\prime}$.

Remark 2. To satisfy the condition $={ }_{E} \circ \rightarrow_{R_{1} \cup R_{2}} \circ=_{E}$ is terminating, it suffices to exhibit an $E$-compatible reduction ordering $>$ such that $l>r$ for any $l \rightarrow$ $r \in R_{1} \cup R_{2}$. In that case, $>$ is defined on terms built over $\Sigma_{1} \cup \Sigma_{2}$.

By Lemma 5 the two assumptions of Lemma 4 can be satisfied, and this leads to a hierarchical unification procedure for the combined TRS. In the following, we consider a notion of decreasingness in order to study the termination of this unification procedure.

Definition 3 (Decreasingness). Consider a complexity measure defined as a mapping $C$ from separate forms to natural numbers. An $H_{E}(U)$ inference system is said to be $C$-decreasing if for any separate form $G \cup G_{0}$ we have that (1) for any $G^{\prime}$ such that $G \cup G_{0} \vdash_{U} G^{\prime} \cup G_{0}$, the separate form of $G^{\prime} \cup G_{0}$ does not increase $C$; (2) for any $G_{0}^{\prime}$ such that $G \cup G_{0} \vdash_{\text {Solve }} G \cup G_{0}^{\prime}$, then either the separate form of $G \cup G_{0}^{\prime}$ is in normal form w.r.t $H_{E}(U)$, or it decreases $C$. 
Consequently, $H_{E}(U)$ is terminating if there exists some $C$ such that $H_{E}(U)$ is $C$-decreasing.

Theorem 1. Assume $E$ is a subterm collapse-free theory such that an E-unification algorithm is known, and $C$ is a complexity measure defined on separate forms. Let $\left(R_{1}, E\right)$ and $\left(R_{2}, E\right)$ be two E-constructed TRSs sharing only symbols in $E$ such that, for $i=1,2,\left(R_{i}, E\right)$ is layer-preserving, and $R_{i} \cup E$ admits a $C$-decreasing unification algorithm of the form $H_{E}\left(U_{i}\right)$. If $={ }_{E} \circ \rightarrow_{R_{1} \cup R_{2}} \circ=_{E}$ is terminating, then $\left(R_{1} \cup R_{2}, E\right)$ is an E-constructed TRS such that $\left(R_{1} \cup R_{2}, E\right)$ is layer-preserving, and $R_{1} \cup R_{2} \cup E$ admits a $C$-decreasing unification algorithm of the form $H_{E}\left(U_{1} \cup U_{2}\right)$.

Proof. $\left(R_{1} \cup R_{2}, E\right)$ is layer-preserving by Lemma 5 . In addition, a $\Sigma_{1} \backslash \Sigma_{0}$-rooted term cannot be equal to a $\Sigma_{2} \backslash \Sigma_{0}$-rooted term modulo $R_{1} \cup R_{2} \cup E$. Applying Lemma $4 H_{E}\left(U_{1} \cup U_{2}\right)$ provides a sound and complete $R_{1} \cup R_{2} \cup E$-unification procedure. Moreover, $H_{E}\left(U_{1} \cup U_{2}\right)$ is $C$-decreasing and so it is terminating.

Example 4. Consider the theories $\mathcal{E}_{A C}$ and $\mathcal{F}_{A C}$ introduced in Proposition 3 and the corresponding hierarchical unification algorithms $H_{A C}\left(U_{\mathcal{E}}\right)$ and $H_{A C}\left(U_{\mathcal{F}}\right)$ where the mutation rules defining $U_{\mathcal{E}}$ and $U_{\mathcal{F}}$ can be found in 15. Let $S V C$ be the complexity measure defined as follows: given an $R \cup E$-unification problem in separate form $G \cup G_{0}, S V C\left(G \cup G_{0}\right)$ is the number of equivalence classes of variables shared by $G$ and $G_{0}$ that are variables abstracting $\Sigma \backslash \Sigma_{0}$-rooted terms.

Let us now check that the unification algorithms $H_{A C}\left(U_{\mathcal{E}}\right)$ and $H_{A C}\left(U_{\mathcal{F}}\right)$ are both $S V C$-decreasing. On the one hand, it is routine to verify that any (mutation) rule in $U_{\mathcal{E}}$ (resp., $U_{\mathcal{F}}$ ) does not lead, via a further possible application of VA, to new shared variables which are abstracting $\Sigma \backslash \Sigma_{0}$-rooted terms. Hence, the rules in $U_{\mathcal{E}}$ (resp., $U_{\mathcal{F}}$ ) cannot increase $S V C$. On the other hand, Solve leads to either a normal form w.r.t $H_{A C}\left(U_{\mathcal{E}}\right)$ (resp., $H_{A C}\left(U_{\mathcal{F}}\right)$ ), or it generates some equality $x=?$ ? $y$ between variables $x$ and $y$ for which there are $\Sigma \backslash \Sigma_{0^{-}}$ equations $x={ }^{?} s$ and $y={ }^{?} t$ in $G$. In the last case, the respective equivalence classes of $x$ and $y$ are merged into a single one by applying Solve and so, Solve strictly decreases $S V C$. By Theorem 1 we get that $\mathcal{E}_{A C} \cup \mathcal{F}_{A C}$ admits a $S V C$-decreasing unification algorithm of the form $H_{A C}\left(U_{\mathcal{E}} \cup U_{\mathcal{F}}\right)$. Notice this means that we can use the termination strategy used in the individual $H_{A C}\left(U_{\mathcal{E}}\right)$ and $H_{A C}\left(U_{\mathcal{F}}\right)$ algorithms to obtain a termination strategy for the hierarchical combined algorithm, $H_{A C}\left(U_{\mathcal{E}} \cup U_{\mathcal{F}}\right)$. We suspect that this complexity measure, $S V C$, could be useful for proving termination in other theories.

To conclude this section, let us mention the problem of combining two copies of the same $E$-constructed layer-preserving TRS, provided that only the symbols in $E$ are possibly shared. In that very particular case, layer-preservingness is sufficient and there is no need to find a decreasing complexity measure.

Theorem 2. Consider $(R, E)$ is an E-constructed layer-preserving TRS over the signature $\Sigma$ such that $R \cup E$ admits a unification algorithm of the form $H_{E}(U)$. Let $\left(R^{\prime}, E\right)$ be a copy of $(R, E)$ obtained by renaming the $\Sigma \backslash \Sigma_{0}$-symbols. 
Then, $\left(R \cup R^{\prime}, E\right)$ is an E-constructed layer-preserving TRS such that $R \cup R^{\prime} \cup E$ admits a unification algorithm of the form $H_{E}\left(U \cup U^{\prime}\right)$, where $U^{\prime}$ is obtained from $U$ by applying the same renaming as the one defining $\left(R^{\prime}, E\right)$.

Proof. Consider the morphism $\iota$ replacing each symbol $f^{\prime} \in \Sigma^{\prime} \backslash \Sigma_{0}$ by the corresponding function symbol $f \in \Sigma \backslash \Sigma_{0}$. For any terms $s, t, s={ }_{E} \circ \rightarrow_{R \cup R^{\prime}} \circ=_{E} t$ implies $\iota(s)=_{E} \circ \rightarrow_{R} \circ=_{E} \iota(t)$. Thus, $=_{E} \circ \rightarrow_{R} \circ=_{E}$ is terminating implies $=_{E} \circ \rightarrow R \cup R^{\prime} \circ=_{E}$ is terminating. By Lemmas 5 and $4 .\left(R \cup R^{\prime}, E\right)$ is an $E$-constructed layer-preserving TRS and $H_{E}\left(U \cup U^{\prime}\right)$ is a sound and complete $R \cup R^{\prime} \cup E$-unification procedure. For each inference $P \vdash_{H_{E}\left(U \cup U^{\prime}\right)} Q$, there exists an inference $\iota(P) \vdash_{H_{E}(U)} \iota(Q)$. Thus, the termination w.r.t $H_{E}(U)$ implies the termination w.r.t $H_{E}\left(U \cup U^{\prime}\right)$.

Example 5. Consider the two E-constructed layer-preserving TRSs $\left(R_{\mathcal{D}}, E\right)$ and $\left(R_{\mathcal{D} 1}, E\right)$ defined in Proposition 2, and their copies $R_{\mathcal{D}}^{\prime}=\left\{h^{\prime}(x * y) \rightarrow h^{\prime}(x) *\right.$ $\left.h^{\prime}(y)\right\}$ and $R_{\mathcal{D} 1}^{\prime}=\left\{f^{\prime}(x * y, z) \rightarrow f^{\prime}(x, z) * f^{\prime}(y, z)\right\}$. The theories $R_{\mathcal{D}} \cup E$ and $R_{\mathcal{D} 1} \cup E$ admit unification algorithms of the form $H_{E}\left(U_{\mathcal{D}}\right)$ and $H_{E}\left(U_{\mathcal{D} 1}\right)$, respectively. By Theorem $2, R_{\mathcal{D}} \cup R_{\mathcal{D}}^{\prime} \cup E$ and $R_{\mathcal{D} 1} \cup R_{\mathcal{D} 1}^{\prime} \cup E$ admit unification algorithms of the form $H_{E}\left(U_{\mathcal{D}} \cup U_{\mathcal{D}}^{\prime}\right)$ and $H_{E}\left(U_{\mathcal{D} 1} \cup U_{\mathcal{D} 1}^{\prime}\right)$, respectively.

\subsection{Combining Forward-Closed E-Constructed TRSs}

The union of two forward-closed $E$-constructed TRSs remains a forward-closed $E$ constructed TRS. Thus, a hierarchical unification algorithm can be constructed in a modular way in unions of forward-closed $E$-constructed TRSs.

Theorem 3. Assume $E$ is a regular and collapse-free theory such that an $E$ unification algorithm is known. Let $\left(R_{1}, E\right)$ and $\left(R_{2}, E\right)$ be two forward-closed E-constructed TRSs sharing only symbols in E. Then $R_{1} \cup R_{2} \cup E$ admits a unification algorithm of the form $H_{E}\left(B S M_{R_{1}} \cup B S M_{R_{2}}\right)$.

Proof. $\left(R_{1} \cup R_{2}, E\right)$ is a forward-closed $E$-constructed TRS, and so by Lemma 3 , $R_{1} \cup R_{2} \cup E$ admits a unification algorithm of the form $H_{E}\left(B S M_{R_{1} \cup R_{2}}\right)$, which coincides with $H_{E}\left(B S M_{R_{1}} \cup B S M_{R_{2}}\right)$.

In the following, we investigate the case where $E$ already admits a hierarchical unification algorithm of the form $H_{E^{\prime}}\left(U^{\prime}\right)$ for a subtheory $E^{\prime}$ of $E$, like in Example 3 where $E$ has a hierarchical unification algorithm of the form $H_{E^{\prime}}\left(U^{\prime}\right)$ for $E^{\prime}=\emptyset$. In that case, we can consider the following compositionality lemma:

Lemma 6. Let $(R, E)$ be an E-constructed TRS such that $R \cup E$ admits a unification algorithm of the form $H_{E}(U)$, and $E$ admits a unification algorithm of the form $H_{E^{\prime}}\left(U^{\prime}\right)$, where $E^{\prime}$ is a subtheory of $E$. Then $R \cup E$ also admits a unification algorithm of the form $H_{E^{\prime}}\left(U \cup U^{\prime}\right)$.

Proof. Consider $\Sigma^{\prime}=\Sigma_{0}$ and $E$ is a $\Sigma^{\prime}$-theory of the form $E=F^{\prime} \cup E^{\prime}$. Assume $R \cup E$ (resp., $F^{\prime} \cup E^{\prime}$ ) has a unification algorithm of the form $H_{E}(U)$ (resp., $H_{E^{\prime}}\left(U^{\prime}\right)$ ), where $U$ (resp., $U^{\prime}$ ) is sound, complete, and parameterized by some 
finite set $S$ (resp., $S^{\prime}$ ) of $R \cup E$-equalities (resp., $F^{\prime} \cup E^{\prime}$-equalities) such that the soundness of each inference $\vdash_{U}\left(\right.$ resp., $\left.\vdash_{U^{\prime}}\right)$ follows from at most one equality in $S$ (resp., $S^{\prime}$ ).

Since $E$-unification is complete for solving the $\Sigma^{\prime}$-fragment of $R \cup E$-unification, $U^{\prime}$ is also sound and complete for $R \cup F^{\prime} \cup E^{\prime}$. Hence, the inference system $U \cup U^{\prime}$ is sound and complete. Moreover, $S \cup S^{\prime}$ is a finite set of $R \cup F^{\prime} \cup E^{\prime}$ equalities such that the soundness of each inference $\vdash_{U \cup U^{\prime}}$ follows from at most one equality in $S \cup S^{\prime}$.

Since $E$-unification is complete for solving the $\Sigma^{\prime}$-fragment of $R \cup E$-unification and $E^{\prime}$-unification is complete for solving the $\Sigma_{0}^{\prime}$-fragment of $E$-unification, we have that $E^{\prime}$ is also complete for solving the $\Sigma_{0}^{\prime}$-fragment of $R \cup F^{\prime} \cup E^{\prime}$ unification.

Consequently, $E^{\prime}, R \cup F^{\prime} \cup E^{\prime}$ and $U \cup U^{\prime}$ satisfy all the assumptions of Definition 1, and so $H_{E^{\prime}}\left(U \cup U^{\prime}\right)$ is well-defined. Since $H_{E^{\prime}}\left(U \cup U^{\prime}\right)$ corresponds to an "unfolding" of $H_{E}(U)$, it is terminating, sound and complete, just like $H_{E}(U)$. Thus, $H_{E^{\prime}}\left(U \cup U^{\prime}\right)$ is a unification algorithm for $R \cup E=R \cup F^{\prime} \cup E^{\prime}$.

Example 6. (Example 3 continued) $R \cup E$ admits a unification algorithm of the form $H_{\emptyset}\left(B S M_{R} \cup U^{\prime}\right)$ where $H_{\emptyset}\left(U^{\prime}\right)$ is a hierarchical $E$-unification algorithm.

Example 7. Let us consider a theory used in practice to model a group messaging protocol [9]. For this protocol, the theory modeling the intruder can be defined [27] as a combination $R_{E N C} \cup K$ where $K=\left\{k \operatorname{keyexch}\left(x, p k\left(x^{\prime}\right), y, p k\left(y^{\prime}\right)\right)=\right.$ $\left.k e y e x c h\left(x^{\prime}, p k(x), y^{\prime}, p k(y)\right)\right\}$ and $\left(R_{E N C}, K\right)$ is the forward-closed $K$-constructed TRS where

$$
R_{E N C}=\left\{\begin{aligned}
\operatorname{adec}(\operatorname{aenc}(m, p k(s k)), s k) & \rightarrow m \\
\operatorname{getmsg}(\operatorname{sign}(m, s k)) & \rightarrow m \\
\operatorname{checksign}(\operatorname{sign}(m, \operatorname{sk}), m, p k(s k)) & \rightarrow o k \\
\operatorname{sdec}(\operatorname{senc}(m, k), k) & \rightarrow m
\end{aligned}\right\}
$$

$K$ is a theory closed by paramodulation and so $K$-unification is finitary 23 . By Lemma 3, $R_{E N C} \cup K$ has a hierarchical unification algorithm of the form $H_{K}\left(B S M_{R_{E N C}}\right)$. The mutation-based unification algorithm known for theories closed by paramodulation [23] can be reworded as a hierarchical unification algorithm, of the form $H_{\emptyset}\left(U_{K}\right)$ for $K$. By Lemma $6, H_{\emptyset}\left(B S M_{R_{E N C}} \cup U_{K}\right)$ is another $R_{E N C} \cup K$-unification algorithm.

Applying Lemma 6, we can easily obtain a hierarchical unification algorithm for a forward-closed $E$-constructed TRS combined with a regular and collapsefree $E$-constructed TRS.

Lemma 7. Assume $E$ is a regular and collapse-free theory such that an $E$ unification algorithm is known. Let $\left(R_{1}, E\right)$ and $\left(R_{2}, E\right)$ be two E-constructed TRSs sharing only symbols in $E$ such that $\left(R_{1}, E\right)$ is forward-closed, and $R_{2} \cup E$ is a regular and collapse-free theory $E_{2}$ admitting a unification algorithm of the form $H_{E}\left(U_{2}\right)$. Then $\left(R_{1}, E_{2}\right)$ is a forward-closed $E_{2}$-constructed TRS and $R_{1} \cup E_{2}$ admits a unification algorithm of the form $H_{E}\left(B S M_{R_{1}} \cup U_{2}\right)$. 
Proof. $\left(R_{1}, E_{2}\right)$ is forward-closed because $\left(R_{1}, E\right)$ is forward-closed and the equational theory $=_{E}$ coincides with $={ }_{E_{2}}$ on $\Sigma_{1}$-terms. By Lemma 3, $R_{1} \cup E_{2}$ admits a unification algorithm of the form $H_{E_{2}}\left(B S M_{R_{1}}\right)$. According to Lemma $6, R_{1} \cup E_{2}$ also admits a unification algorithm of the form $H_{E}\left(B S M_{R_{1}} \cup U_{2}\right)$.

Example 8. Let $(R, A C(\circledast))$ be a forward-closed $A C(\circledast)$-constructed TRS such that $\circledast$ is the only function symbol shared by $R \cup A C(\circledast)$ and $\mathcal{E}_{A C}$ (resp., $\mathcal{F}_{A C}$ ). By Lemma 7, $R \cup \mathcal{E}_{A C}$ (resp., $R \cup \mathcal{F}_{A C}$ ) admits a unification algorithm of the form $H_{A C}\left(B S M_{R} \cup U_{\mathcal{E}}\right)$ (resp., $H_{A C}\left(B S M_{R} \cup U_{\mathcal{F}}\right)$ ). According to Example 4. $\mathcal{E}_{A C} \cup \mathcal{F}_{A C}$ admits a unification algorithm of the form $H_{A C}\left(U_{\mathcal{E}} \cup U_{\mathcal{F}}\right)$. Then, by Lemma 7, $R \cup \mathcal{E}_{A C} \cup \mathcal{F}_{A C}$ admits a unification algorithm of the form $H_{A C}\left(B S M_{R} \cup U_{\mathcal{E}} \cup U_{\mathcal{F}}\right)$.

\section{Conclusion}

We have introduced a hierarchical unification framework as a generic tool to construct unification procedures for (combined) equational theories defined by $E$-constructed TRSs. We have presented new combination results for the simplest case of subterm collapse-free theories, and a natural follow-up would be to study the case of regular and collapse-free theories. A challenging future work is to investigate the general case of arbitrary theories.

Hierarchical unification allows us to handle syntactic theories $R \cup E$ while the $E$-unification algorithm can be arbitrary. According to this observation, we plan to study a weakening of syntacticness, in order to allow theories $R \cup E$ that are just syntactic modulo $E$.

We have also begun the implementation of the above hierarchical combination procedure. To begin with, we are using $E=A C$ as the background theory. However, we will explore expanding this to additional equational theories. In the short term, we plan to experiment the use of our variant-free hierarchical unification procedures (e.g., the ones introduced in Examples 3 and 7) as an alternative to variant-based unification procedures in modern protocol verification tools 6 6 18 25. In the long term, we want to promote the use of non-disjoint combination procedures [16] and mutation-based procedures [17] in protocol verification tools, targeting unification problems as well as some decision problems related to the knowledge of an intruder, such as intruder deduction (a reachability problem) and indistinguishability (an equivalence problem) [18. The goal is

to improve automation of verification methods when theories share for instance $A C$ symbols.

\section{References}

1. Abadi, M., Cortier, V.: Deciding knowledge in security protocols under equational theories. Theor. Comput. Sci. 367(1-2), 2-32 (2006)

2. Baader, F., Nipkow, T.: Term rewriting and all that. Cambridge University Press, New York, NY, USA (1998) 
3. Baader, F., Schulz, K.U.: Unification in the union of disjoint equational theories: Combining decision procedures. Journal of Symbolic Computation 21(2), 211 243 (1996)

4. Baader, F., Snyder, W.: Unification theory. In: Robinson, J.A., Voronkov, A. (eds.) Handbook of Automated Reasoning, pp. 445-532. Elsevier and MIT Press (2001)

5. Basin, D.A., Mödersheim, S., Viganò, L.: An on-the-fly model-checker for security protocol analysis. In: Snekkenes, E., Gollmann, D. (eds.) Computer Security ESORICS 2003, 8th European Symposium on Research in Computer Security, Gjøvik, Norway, October 13-15, 2003, Proceedings. Lecture Notes in Computer Science, vol. 2808, pp. 253-270. Springer (2003)

6. Blanchet, B.: Modeling and verifying security protocols with the Applied Pi calculus and ProVerif. Foundations and Trends in Privacy and Security 1(1-2), 1-135 (2016)

7. Bouchard, C., Gero, K.A., Lynch, C., Narendran, P.: On forward closure and the finite variant property. In: Fontaine, P., Ringeissen, C., Schmidt, R.A. (eds.) Frontiers of Combining Systems - 9th International Symposium, FroCoS 2013, Nancy, France, September 18-20, 2013. Proceedings. Lecture Notes in Computer Science, vol. 8152, pp. 327-342. Springer (2013)

8. Ciobâcă, S., Delaune, S., Kremer, S.: Computing knowledge in security protocols under convergent equational theories. J. Autom. Reasoning 48(2), 219-262 (2012)

9. Cohn-Gordon, K., Cremers, C., Garratt, L., Millican, J., Milner, K.: On ends-toends encryption: Asynchronous group messaging with strong security guarantees. In: Lie, D., Mannan, M., Backes, M., Wang, X. (eds.) Proceedings of the 2018 ACM SIGSAC Conference on Computer and Communications Security, CCS 2018, Toronto, ON, Canada, October 15-19, 2018. pp. 1802-1819. ACM (2018)

10. Comon, H., Haberstrau, M., Jouannaud, J.: Syntacticness, cycle-syntacticness, and shallow theories. Inf. Comput. 111(1), 154-191 (1994)

11. Comon-Lundh, H., Delaune, S.: The finite variant property: How to get rid of some algebraic properties. In: Giesl, J. (ed.) Rewriting Techniques and Applications. Lecture Notes in Computer Science, vol. 3467, pp. 294-307. Springer (2005)

12. Durán, F., Eker, S., Escobar, S., Martí-Oliet, N., Meseguer, J., Talcott, C.L.: Built-in variant generation and unification, and their applications in Maude 2.7. In: Olivetti, N., Tiwari, A. (eds.) Automated Reasoning - 8th International Joint Conference, IJCAR 2016, Coimbra, Portugal, June 27 - July 2, 2016, Proceedings. Lecture Notes in Computer Science, vol. 9706, pp. 183-192. Springer (2016)

13. Eeralla, A.K., Erbatur, S., Marshall, A.M., Ringeissen, C.: Rule-based unification in combined theories and the finite variant property. In: Martín-Vide, C., Okhotin, A., Shapira, D. (eds.) Language and Automata Theory and Applications - 13th International Conference, LATA 2019, St. Petersburg, Russia, March 26-29, 2019, Proceedings. Lecture Notes in Computer Science, vol. 11417, pp. 356-367. Springer (2019)

14. Erbatur, S., Kapur, D., Marshall, A.M., Narendran, P., Ringeissen, C.: Hierarchical combination. In: Bonacina, M.P. (ed.) Automated Deduction - CADE-24 - 24th International Conference on Automated Deduction, Lake Placid, NY, USA, June 914, 2013. Proceedings. Lecture Notes in Computer Science, vol. 7898, pp. 249-266. Springer (2013)

15. Erbatur, S., Marshall, A.M., Kapur, D., Narendran, P.: Unification over distributive exponentiation (sub)theories. Journal of Automata, Languages and Combinatorics (JALC) 16(2-4), 109-140 (2011) 
16. Erbatur, S., Marshall, A.M., Ringeissen, C.: Notions of knowledge in combinations of theories sharing constructors. In: de Moura, L. (ed.) Automated Deduction CADE 26 - 26th International Conference on Automated Deduction, Gothenburg, Sweden, August 6-11, 2017, Proceedings. Lecture Notes in Computer Science, vol. 10395, pp. 60-76. Springer (2017)

17. Erbatur, S., Marshall, A.M., Ringeissen, C.: Computing knowledge in equational extensions of subterm convergent theories. Math. Struct. Comput. Sci. 30(6), 683709 (2020)

18. Escobar, S., Meadows, C.A., Meseguer, J.: Maude-NPA: Cryptographic protocol analysis modulo equational properties. In: Aldini, A., Barthe, G., Gorrieri, R. (eds.) Foundations of Security Analysis and Design, Tutorial Lectures. Lecture Notes in Computer Science, vol. 5705, pp. 1-50. Springer (2007)

19. Escobar, S., Sasse, R., Meseguer, J.: Folding variant narrowing and optimal variant termination. J. Log. Algebr. Program. 81(7-8), 898-928 (2012)

20. Jouannaud, J., Kirchner, H.: Completion of a set of rules modulo a set of equations. SIAM J. Comput. 15(4), 1155-1194 (1986)

21. Kim, D., Lynch, C., Narendran, P.: Reviving basic narrowing modulo. In: Herzig, A., Popescu, A. (eds.) Frontiers of Combining Systems - 12th International Symposium, FroCoS, London, UK, Proceedings. Lecture Notes in Computer Science, vol. 11715, pp. 313-329. Springer (Sep 2019)

22. Kirchner, C., Klay, F.: Syntactic theories and unification. In: Logic in Computer Science, 1990. LICS '90, Proceedings, Fifth Annual IEEE Symposium on Logic in Computer Science. pp. 270-277 (Jun 1990)

23. Lynch, C., Morawska, B.: Basic syntactic mutation. In: Voronkov, A. (ed.) Automated Deduction - CADE-18, 18th International Conference on Automated Deduction, Copenhagen, Denmark, July 27-30, 2002, Proceedings. Lecture Notes in Computer Science, vol. 2392, pp. 471-485. Springer (2002)

24. Marshall, A.M., Meadows, C.A., Narendran, P.: On unification modulo one-sided distributivity: Algorithms, variants and asymmetry. Logical Methods in Computer Science 11(2) (2015)

25. Meier, S., Schmidt, B., Cremers, C., Basin, D.A.: The TAMARIN prover for the symbolic analysis of security protocols. In: Sharygina, N., Veith, H. (eds.) Computer Aided Verification - 25th International Conference, CAV 2013, Saint Petersburg, Russia, July 13-19, 2013. Proceedings. Lecture Notes in Computer Science, vol. 8044, pp. 696-701. Springer (2013)

26. Meseguer, J.: Variant-based satisfiability in initial algebras. Sci. Comput. Program. 154, 3-41 (2018)

27. Nguyen, K.: Formal verification of a messaging protocol, work done under the supervision of Vincent Cheval and Véronique Cortier

28. Nipkow, T.: Proof transformations for equational theories. In: Logic in Computer Science, 1990. LICS '90, Proceedings, Fifth Annual IEEE Symposium on Logic in Computer Science. pp. 278-288 (Jun 1990)

29. Schmidt-Schauß, M.: Unification in a combination of arbitrary disjoint equational theories. Journal of Symbolic Computation 8, 51-99 (July 1989)

30. Tidén, E., Arnborg, S.: Unification problems with one-sided distributivity. Journal of Symbolic Computation 3(1/2), 183-202 (1987)

31. Yelick, K.A.: Unification in combinations of collapse-free regular theories. Journal of Symbolic Computation 3(1-2), 153-181 (1987) 